\title{
Simulare per RI_Connettere. VR per i disturbi dello spettro autistico
}

\author{
Mara Capone \\ Emanuela Lanzara
}

\section{Abstract}

I disturbi ASD sono sindromi neuro comportamentali complessi; viaggiare, stare in posti affollati, fare una visita medica, approcciare un coetaneo, andare all'Università, sono solo alcuni esempi che possono generare condizioni di ansia e che, spesso, dis_connettono il soggetto dalla vita reale. L'obiettivo della ricerca è quello di sperimentare l'utilizzo di tecnologie innovative per favorire gli scambi relazionali e funzionali tra persone con autismo e persone neuro tipiche, per favorire l'autonomia individuale delle persone con autismo, nella gestione del quotidiano e nella comunicazione sociale, soprattutto nell'età evolutiva e adulta. Nell'ambito di un progetto di ricerca è in corso di sperimentazione la realizzazione di un'app che sarà realizzata da un team multidisciplinare composto da esperti, neuropsichiatri, psicologi clinici, informatici e architetti. L'app simula spazi e situazioni, 'semplifica' la visualizzazione degli ambienti e 'personalizza', con diversi livelli di complessità e interattività, adattandosi ad utenti con differenti livelli di abilità intellettiva e linguistica. Nell'ambito delle nostre specifiche competenze sono oggetto di studio le diverse modalità di semplificazione dello spazio simulato e le diverse modalità di interazione, dal semplice video (livello 0), alle panoramiche $360^{\circ}$ (livelli I e 2), fino alla sperimentazione dell'utilizzo della VR e l'utilizzo di wearable technology.

Parole chiave

VRVirtual Reality, autismo, User Experience, immagini immersive, video modeling, wearable technology.

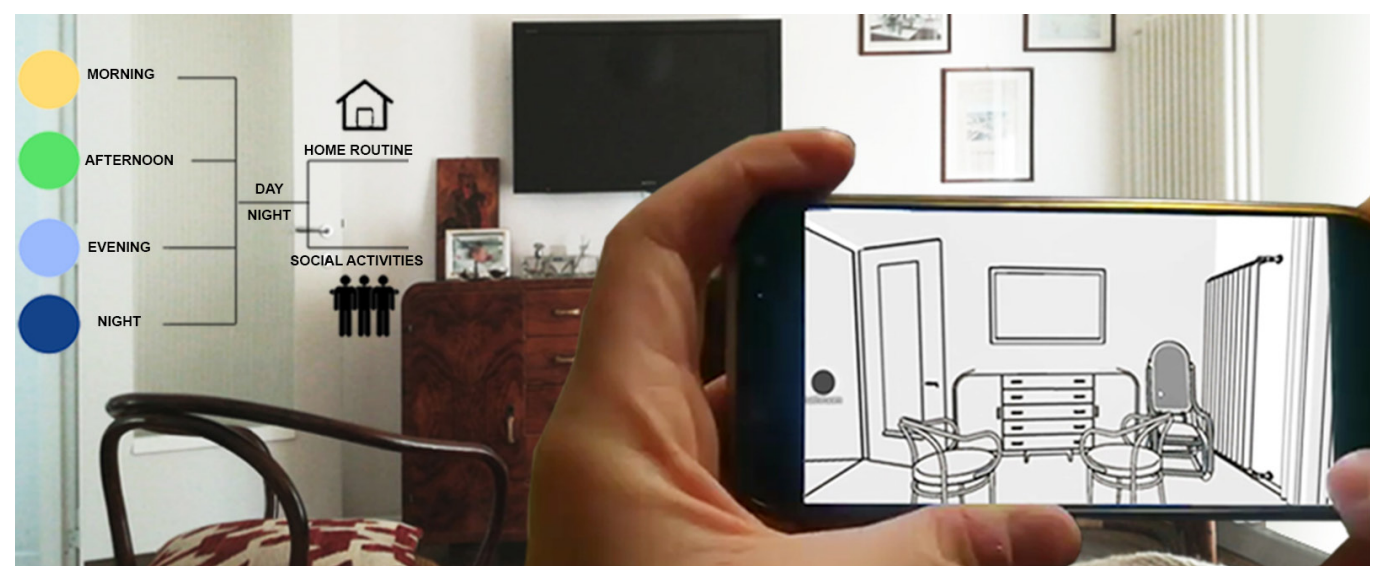




\section{Introduzione}

I disturbi ASD sono sindromi neuro comportamentali complessi, frequentemente associati a comorbidità. Circa il 50\% degli adulti con ASD sperimenta livelli di ansia che incidono sulla vita di tutti i giorni. Recenti studi [Kristy 20 I 8] sostengono l'importanza di individuare supporti atti a favorire l'adattamento persona-ambiente nei soggetti con ASD. La VR è stata utilizzata con successo per migliorare varie capacità [Maskey 2019], comprensione sociale [Kandalaft et al. 20I3], colloquio di lavoro [Smith et al. 20/4] e può offrire un'alternativa alle solite gerarchie di esposizione utilizzate nella CBT tradizionale. Dall'analisi dei risultati emerge come gli out come di trattamento siano influenzati dal livello di adattamento persona-ambiente e che, considerando la variabilità delle manifestazioni, i supporti dovrebbero essere personalizzati. Viaggiare, stare in posti affollati, fare una visita medica, approcciare un coetaneo, andare all'università, sono solo alcuni esempi che possono generare condizioni di ansia e costituire uno scoglio nella vita quotidiana di un ASD. I protocolli terapeutici applicati alla popolazione generale sono talvolta inefficaci negli ASD, per la difficoltà ad immaginare l'oggetto/situazione per poi passare gradualmente ad un'esposizione in vivo. Uno strumento diVRIVM (Virtual Reality Video Modeling) consente di lavorare sulla simulazione bypassando tale difficoltà.

Sulla base degli studi scientifici che dimostrano l'efficacia di strumenti terapeutici basati sull'utilizzo della $\bigvee R$, il progetto propone un'app utilizzabile con mobile device per la gestione di una comorbidità ad alto impatto su skill di tipo sociale, comunicativo e di autonomia basata sulla simulazione di scenari visualizzabili con diversi livelli di complessità (gamesl astrattalreale) e interattività (videolpanoramiche $360^{\circ} \mathrm{IVRlavatar}$ ) per utenti con differenti livelli di abilità intellettiva e linguistica. Le scene saranno individualizzate in base alle esigenze di ciascun partecipante; uno psicoterapeuta a formazione cognitivo-comportamentale guiderà l'esposizione e applicherà tecniche di CBT per lavorare sul rilassamento, sul condizionamento e sul ricondizionamento allo stimolo avverso seguendo le linee guida nice.

Rispetto alle app attualmente esistenti, l'obiettivo della ricerca è quello di realizzare uno strumento 'adattabile' alle variabili esigenze di adulti con ASD che affronta con particolare attenzione le questioni dell'user experience e della personalizzazione.

L'app sarà il risultato di un lavoro interdisciplinare. II team, composto da architetti esperti di modellazione e gestione di grafica 3D, informatici esperti nel design di sistemi interattivi, clinici esperti dello spettro autistico e una onlus che mette a disposizione una platea di soggetti per la sperimentazione, comprende le competenze necessarie per definire uno strumento in grado di simulare spazi e situazioni in risposta alle specifiche esigenze percettive di adulti con ASD.

Figg. I, 2. Interfacce semplificate per l'App prototipo.
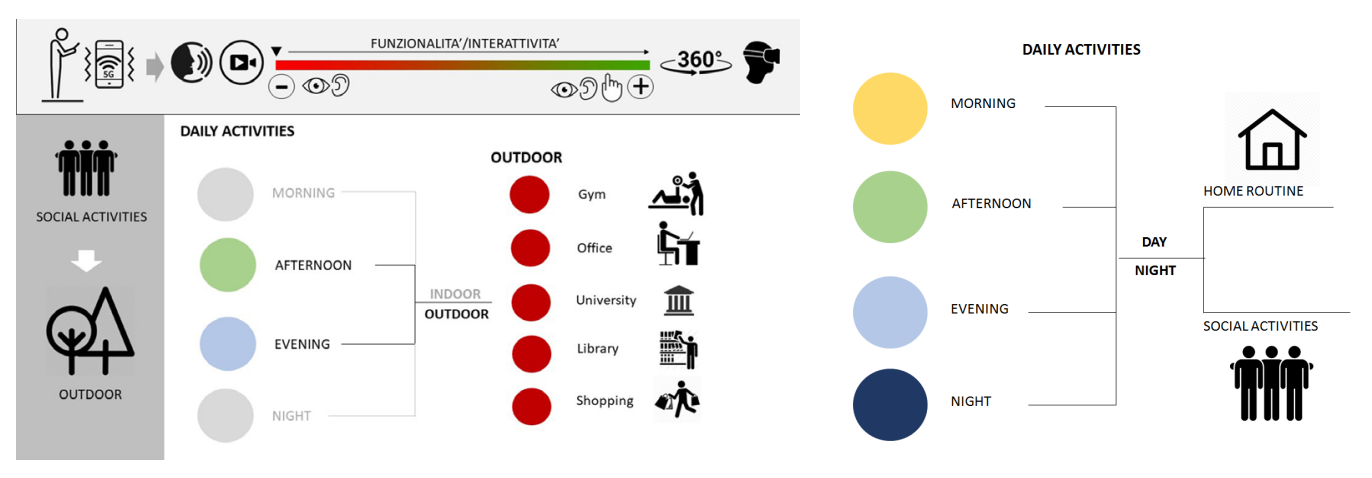

\section{Obiettivi del progetto}

L'app adattabile, personalizzabile, open source, user friendly destinata ad adulti con ASD, alle famiglie e ai terapeuti per migliorare l'adattabilità persona-ambiente, ha come obiettivi:

- favorire l'autonomia personale e la comunicazione sociale, attraverso l'uso combinato di 
VR (Virtual Reality) e VM (Video Modeling), simulando spazi e situazioni per controllare l'esposizione e la gerarchia di stimoli all'ansia in modo sicuro e misurato;

- raggiungere il maggior numero di utenti con uno strumento 'adattabile' alle esigenze di adulti con ASD in funzione della personale abilità e sensibilità sensoriale (Training Emotions in an Adaptable Reality System) e personalizzabile, consentendo la possibilità di passare da un generico ambiente a quello specifico dell'utente.

- condividere la sperimentazione sui 20 adulti coinvolti diffondendo l'app, attraverso network esistenti, e promuovendo incontri reali e virtuali con gli stakeholder.

\section{Tecnologie per RI_Connettere}

La realtà virtuale VR è considerata un emergente approccio terapeutico efficace in diverse aree del campo sanitario. L'utilizzo dellaVR nel trattamento dell'ASD permette di lavorare in un ambiente realistico che può essere adattato alle caratteristiche e capacità del soggetto.

Uno scenario virtuale può essere un ambiente che toglie la pressione dal vivere la realtà reale $(\mathrm{RR})$ che i soggetti con ASD tendono a rifiutare. L'obiettivo della simulazione è quello di acquisire la consapevolezza spaziale per preparare il soggetto ad affrontare una situazione in un dato spazio.

Per realizzare l'app saranno utilizzate le tecnologie più innovative per:

-costruire modelli 3D finalizzati a produrre immagini e video panoramici fruibili interattivamente a $360^{\circ}$ con mobile devices smartphone, tablet o visori VR;

- visualizzare gli ambienti con diversi livelli di complessità (gameslastrattalreale) e interattività (videolpanoramiche $360^{\circ} \mathrm{IVRlavatar}$ ) in funzione dei diversi livelli di funzionalità, minimo (video non interattivi, visualizzazioni minimali, avatar) medio e massimo (visualizzazione 3D immersiva con visore);

-sperimentare l'utilizzo di immagini $360^{\circ}$ per accedere, tramite hotspot, ai video modeling che simulano le azioni che possono aver luogo in un determinato spazio;

- utilizzare la fotomodellazione e le immagini a $360^{\circ}$ realizzabili con apposite videocamere o con smartphone per sostituire, nell'app, ai modelli generici i modelli reali: un bagno-il mio bagno, una palestra-la mia palestra;

- pianificare le azioni della giornata.

Adattabilità, personalizzabilità sono le principali caratteristiche dell'app che rendono lo strumento flessibile, facilmente utilizzabile, gestibile direttamente dalle famiglie, con un minimo training e supporto, che lo rendono replicabile in altre realtà riabilitative/educative/lavorative. II collegamento con banche dati per caricare VM, la possibilità di implementare gli scenari simulati e personalizzare gli spazi rende l'app adattabile a seconda delle esigenze.

L'app prevede la possibilità di personalizzare gli ambienti virtuali, utilizzando le immagini degli ambienti reali degli utenti che il team di progetto provvederà a trattare e ad inserire.

\section{D PECS per la Comunicazione Aumentativa Alternativa (CAA)}

Il livello di semplificazione della realtà è dettato dalle differenti esigenze degli utenti ASD in relazione allo specifico livello funzionale che li caratterizza. Nell'ambito degli studi scientifici dedicati allo sviluppo di sistemi alternativi di linguaggio, il PECS [I] (Picture Exchange Communication System - sistema di comunicazione mediante scambio per immagini) è una strategia per la Comunicazione Aumentativa Alternativa (CAA) relativa alla gestione dei disturbi dello Spettro Autistico. II suo funzionamento è basato sulla differenziazione tra parlare e comunicare, entrambe attività finalizzate a connettere un individuo con l'ambiente che lo circonda, ma con una più ampia possibilità di scelta dello strumento comunicativo (alternativo alla parola) offerta dalla seconda azione [Blondy et al. 1994].

In sintesi, le conoscenze e le tecniche sottese a tale strategia di comunicazione incrementano ed agevolano le modalità di approccio di quegli utenti ASD con difficoltà ad usare i canali trasmissivi che potremmo definire più immediati, tra cui il linguaggio orale e scritto [Rivarola 2009]. 
Fig. 3. Esempio di immagin per la CAA di tipo "widget", ARASAAC e BLISS Le figure riportate sulle tessere mostrano livelli di zione della realtà differenti [Perugini 2019] I simboli [Perugini 2019$]$. I simboli mossono essere utilizzati mediante dispositivi digital o cards stampate e raccote in dei libretti.
Infatti, tale strategia 'alternativa' viene definita 'aumentativa' in quanto non intende sostituire le naturali modalità comunicative ma è finalizzata ad incrementare le stesse mediante differenti forme di comunicazione, tra cui, in particolare, quelle grafiche [2]. In qualità di linguaggio simbolico, esso richiama strumenti e metodologie differenti di rappresentazione. Nella pratica, la comunicazione tramite tale linguaggio prevede la preparazione di numerose carte illustrate raccolte a creare un personale libretto di comunicazione portatile ed utilizzabile in diversi contesti della vita reale domestica e sociale.
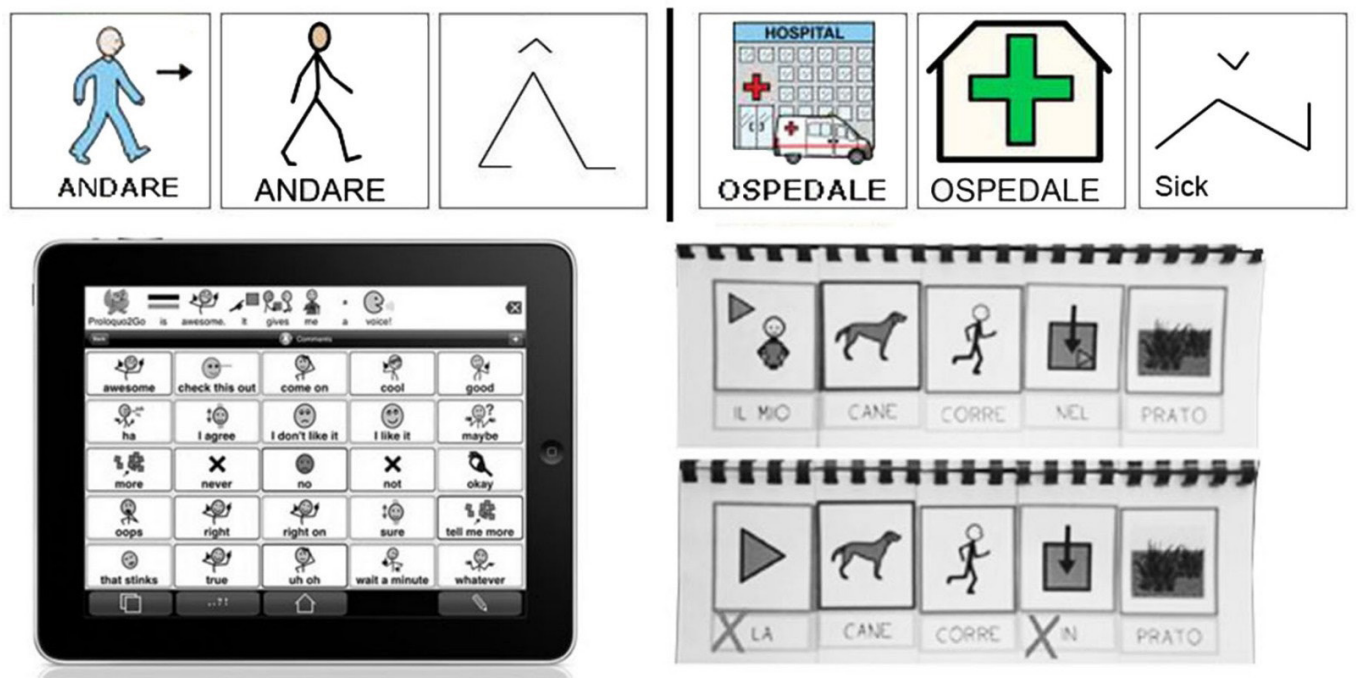

Confermando lo stesso approccio esemplificativo della realtà, lo spazio digitale/virtuale consente di aumentare la dimensione comunicativa di tale supporto o strumento caratterizzato dal susseguirsi di simboli grafici riportati su carta o su supporto digitale per tradurre attività complesse attraverso un codice bidimensionale di segni con immagini, oggetti e contesti tridimensionali navigabili.

Tale passaggio consente all'utente di immergersi in un ambiente interattivo e predittivo delle attività da svolgere in un dato tempo futuro e in determinato ambiente, da solo o in compagnia di altri partecipanti alle attività.

L'ambiente ideale capace di consentire il passaggio dal supporto bidimensionale allo spazio tridimensionale proposto in questo contributo è un'applicazione fruibile attraverso diversi tipi di dispositivi mobili (smartphone, tablet ecc.) ed implementabile con video e simulazioni capaci di configurarsi come l'equivalente tridimensionale e dinamico di tali simboli bidimensionali semplificati.

L'oggetto della semplificazione non è dunque la rappresentazione statica di un'azione ma la modellazione di uno spazio che consenta di simulare tale azione all'interno di una realtà percettiva alternativa più accessibile ad un utente con differenti capacità di interazione. L'app è intesa, in questo senso, come una raccolta virtuale portatile di tutorial utili ad affrontare le azioni quotidiane e ad interagire con gli ambienti nei quali esse si svolgono.

Affinché l'app svolga con successo la propria funzione è necessario disporre di diversi gradi di complessità in base alle caratteristiche cognitive e comunicative dell'utente ASD: la potenza di tali modelli tridimensionali esemplificativi della realtà risiede nel fornire all'utente uno strumento la cui impostazione ed il cui funzionamento siano compatibili con le proprie specifiche possibilità o abilità.

Pertanto, così come il sistema CAA accoppia un'icona esplicativa per ogni attività quotidiana prevista, allo stesso modo l'app accoppierà un icona/oggetto hotspot attivante differenti gradi di semplificazione di uno spazio, un cambio ambiente o un video modeling di una data attività da compiere.

Affinché il processo di semplificazione sia efficace è importante mantenere un buon equilibrio tra completezza dell'informazione e grado di semplificazione. 


\section{SketchUp: cartoon styles per lo sviluppo di un sistema PECS 3D}

Per la realizzazione del'app prototipo, la modellazione degli ambienti che compongono un'abitazione domestica o che rappresentano luoghi di ritrovo comuni (scuola, biblioteca, palestra etc.) è stata realizzata con il software SketchUp.

In passato, importanti esperienze condotte sul campo [3] hanno dimostrato che per alcuni utenti, in particolare quelli non verbali, SketchUp ha rappresentato un efficace mezzo di comunicazione per la condivisione dei pensieri mediante immagini, raggiungendo importanti obiettivi educativi e lavorativi.

La letteratura scientifica (Wright et al. 20II) documenta esiti positivi circa l'utilizzo di questa interfaccia CAD da parte di utenti ASD, tra cui esperienze di modellazione di oggetti semplici bidimensionali (cerchi, quadrati), lo sviluppo di relazioni interpersonali più forti e maggiore fiducia grazie all'utilizzo di una piattaforma capace di mettere in luce abilità di pazienti spesso assopite o latenti [4].

Pertanto, il progetto di comunicazione presentato in questo contributo sceglie SketchUp per l'interfaccia e l'approccio intuitivo alla modellazione (ottimale per un eventuale uso indipendente o per lo sviluppo di un progetto didattico che coinvolga soprattutto utenti ad alto livello di funzionalità) ideale per gestire i livelli di semplificazione degli ambienti e delle attività che caratterizzano la vita quotidiana di un utente generico.

Gli Stili di visualizzazione proposti dal software (Realistic, Shaded e Hidden Line) grazie all'effetto cartoon o sketch che li caratterizza, riprendono le modalità di graficizzazione e consentono la gradualità di semplificazione riscontrabile confrontando le figure dei diversi sistemi utilizzati per le PECS cards che, in tal senso, vengono trasformate in oggetti ed ambienti 3D mediante modalità di rappresentazione basate su un approccio scientifico e consolidato.
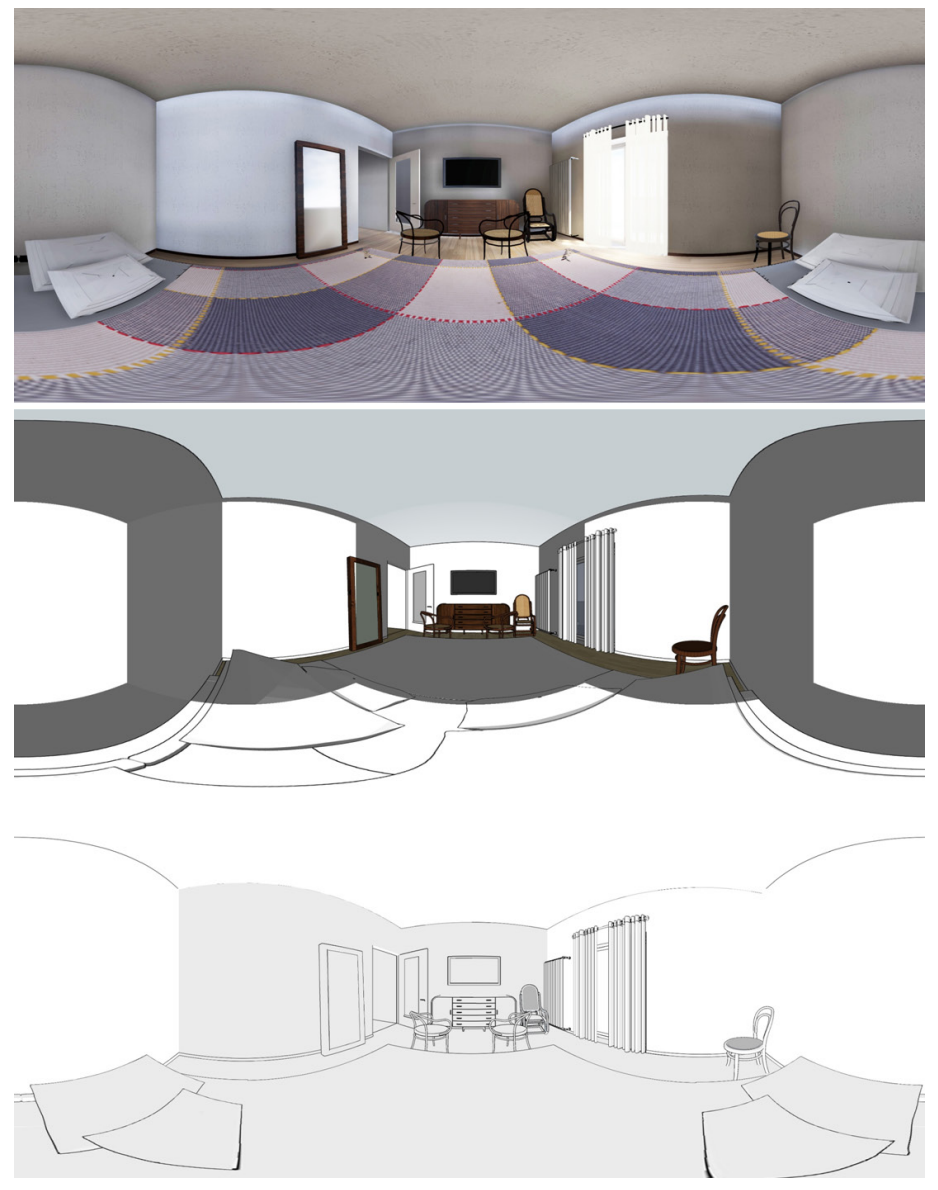


\section{Sistemi di rappresentazione predittiva: dai render/video sferici ai video modeling}

Grazie all'implementazione di appositi plug-in veloci ed intuitivi (e.g. Tour Facil [5]) è possibile esportare, in maniera rapida ed intuitiva, render $360^{\circ}$ degli ambienti modellati direttamente in SketchUp utilizzando gli stili di visualizzazione predefiniti elencati nel paragrafo precedente.

Tale sistema non necessita dell'impostazione del sistema di riferimento prospettico (posizione del punto di vista e profondità del campo visivo) necessaria per generare un render sferico: è sufficiente inquadrare una scena di partenza dell'ambiente che si vuole restituire in modalità 360, utilizzare il plug-in e caricare su un'apposita piattaforma sei viste piane dell'ambiente generate dalla proiezione dello stesso sulle sei facce di un cubo. Per generare un'immagine cubica è necessario utilizzare sei immagini di formato quadrato aventi stesse dimensioni, stesso nome ma suffisso diverso (e.g. prova camera.jpg-Front; prova camera. jpg-Down etc.) per indicarne la reciproca posizione e definire in modo univoco la posizione dell'immagine rispetto alle facce di un cubo (cube maps).

Le panoramiche diventano materiale utilizzabile per simulare con apposite app VR (open source o disponibili in commercio) la percorribilità di un ambiente con livelli di semplificazione differenti.

Fig. 5. Cubic photo o render di un ambiente domestico. L'immagine ealizzata visualizzando l'ambiente bagno modellata in SketchUp con lo stile di visualizzazione hidden line $(\mathrm{b} / \mathrm{n})$, prevede l'inserimento di oggetti scaricabili dalla piattaforma 3D WarehouseSketchUpda evidenziare e specializzare con appositi hotspot collegati ai video modelin corrispondenti alle attivita direttamente relazionabil
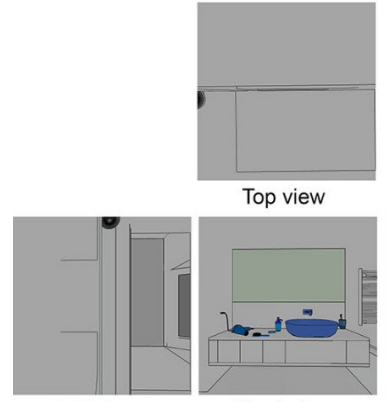

Left view

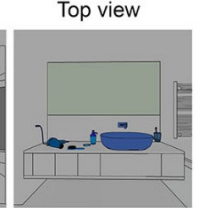

Front view

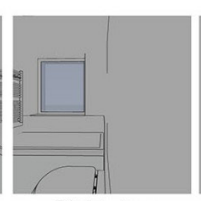

Right view

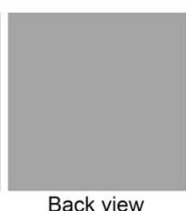

1

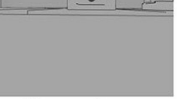

Down view

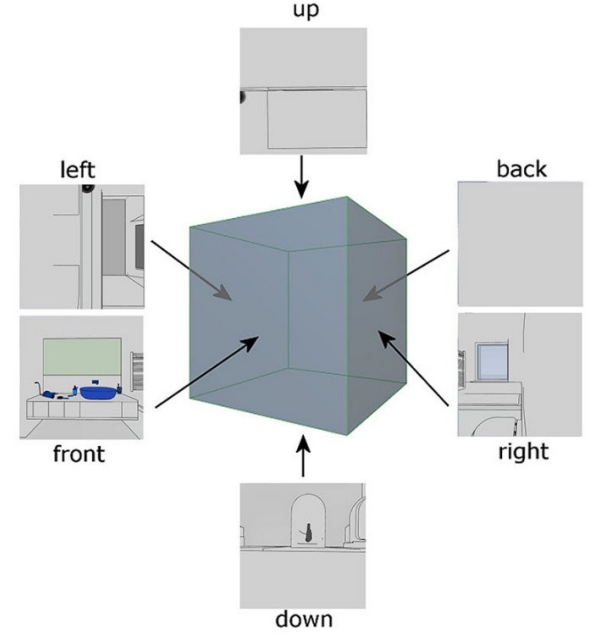

down

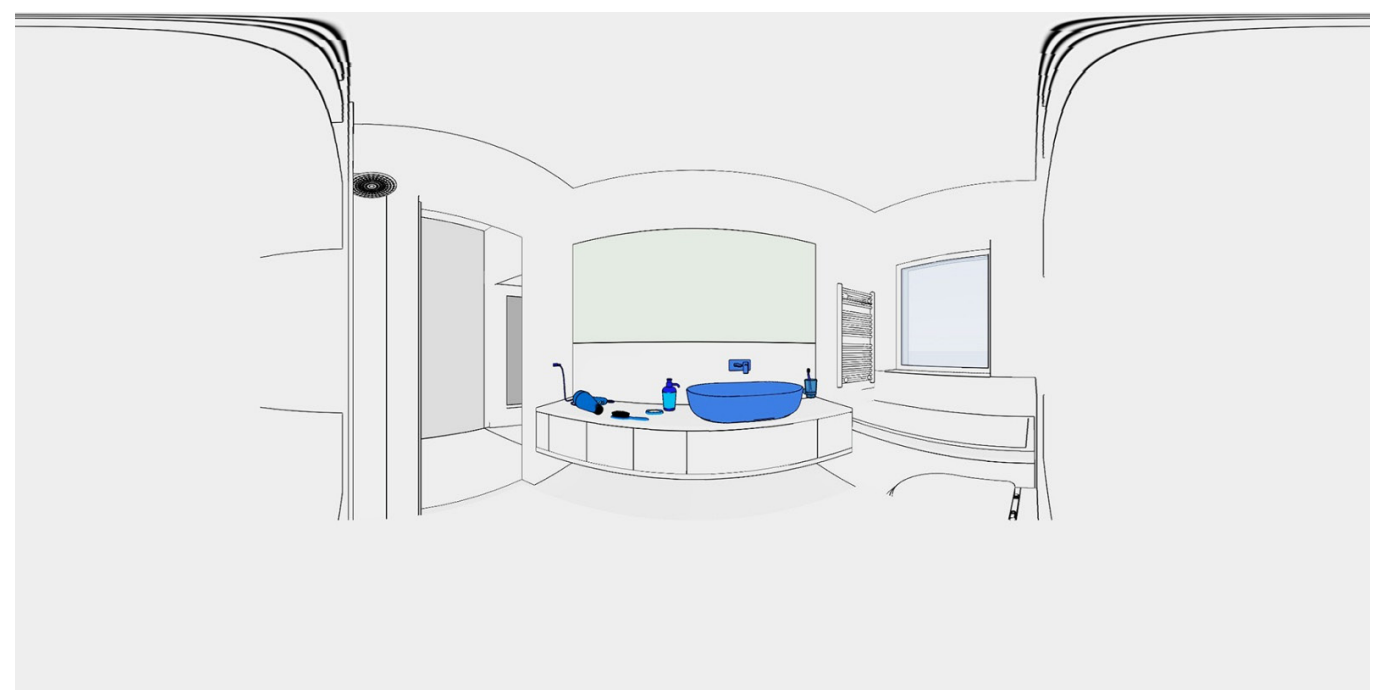


I render sferici o cubici potranno essere fruiti a monitor o con l'utilizzo di un visore in base al livello di funzionalità dell'utente che utilizza l'app. Dopo avere stabilito l'ordine di successione degli ambienti in base alle attività pianificate per la giornata o all'effettiva percorribilità dell'ambiente, è possibile attivare il cambio scena cliccando con il dito o puntando con il visore su una serie di hotspots opportunamente posizionati all'interno delle viste navigabili che aprono quindi le sferiche degli ambienti immediatamente successivi. Gli hotspot consentono anche il passaggio da uno stile di visualizzazione all'altro variando il grado di semplificazione dell'ambiente. La semplicità di modellazione, ma soprattutto la ricca libreria di oggetti modellati disponibili in Sketchup3D Warehouse [6], consentono di caratterizzare gli ambienti con oggetti semplificati che, collegati ad un hotspot posizionato su ciascuno di essi, rappresentano dei bottoni sui quali cliccare per avviare i video modeling la cui azione simulata è intuitivamente collegabile allo specifico oggetto selezionato (e.g.: oggetto phon hotspot - click = video modeling come asciugare i capelli).

Fig. 6. Interfaccia dell'App prototipo per la scelta del livello di visualizzazione in funzione del livello di funzionalità.
Fig. 7. L'utente può passare da una modalità di visualizzazione all'altre anche durante l'esplorazione con le diverse modalità in funzione del livello di interattività utilizzato.

\section{UX DESIGN}

II progetto dell'User Experience prevede una fase di sperimentazione sull'App prototipo con l'obiettivo di definire livelli di semplificazione e di interazione con i contesti simulati adeguati ai diversi livelli di funzionalità. SEMPLIFICARE

L'utente può scegliere la modalità di visualizzazione

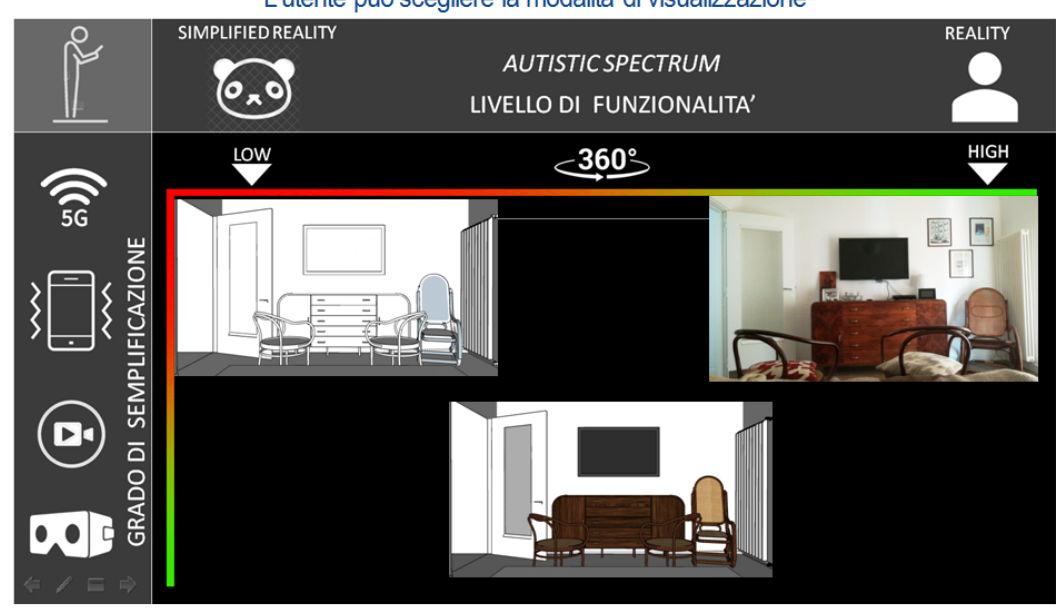

UX DESIGN

II progetto dell'User Experience prevede una fase di sperimentazione sull'App prototipo con l'obiettivo di definire livelli di semplificazione e di interazione con i contesti simulati adeguati ai diversi livelli di funzionalità. SEMPLIFICARE

L'utente può passare da una modalità di visualizzazione all'altra anche durante l'esplorazione utilizzando la funzione touch screen o il sistema di puntatore per VR
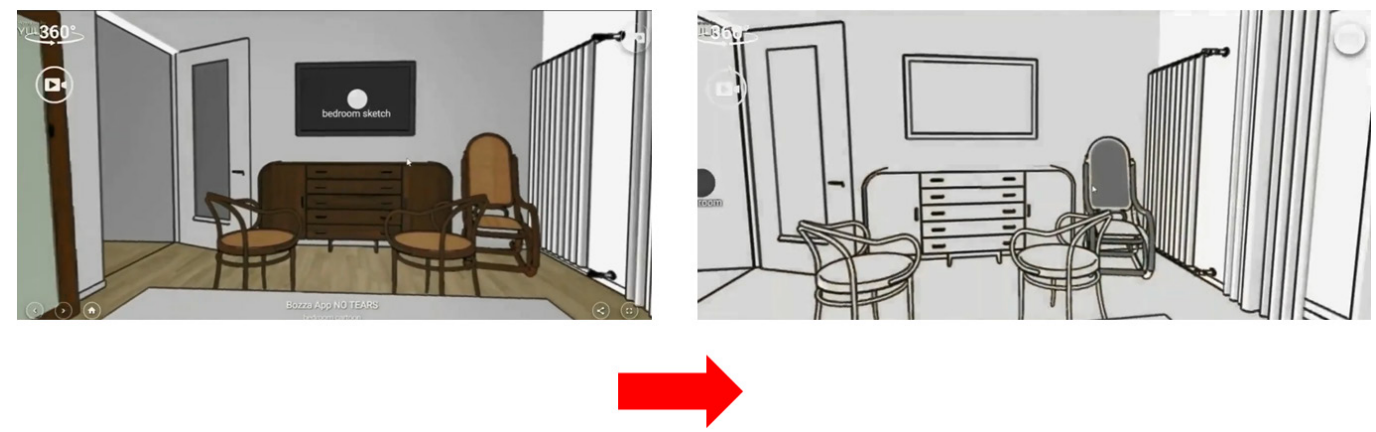
PERSONALIZZARE IL LIVELLO DI INTERATTIVITA'

L'App prevede diversi livelli di complessità e interattività adattandosi ad utenti con differenti livelli di abilità intellettive.

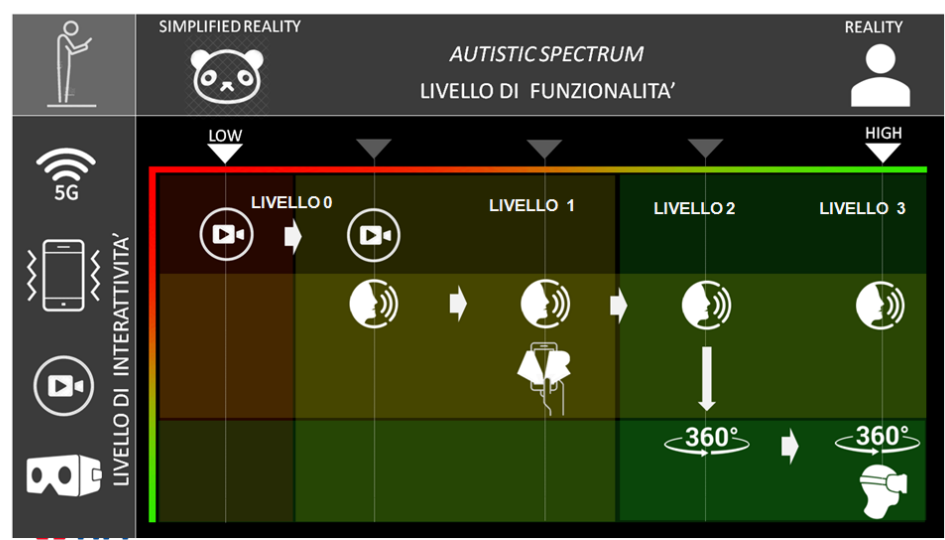

LIVELLOO 0

SOlo VIDEO AUDIO

Lutente non ein grado di interagire autoromamente

LIVELLO 1

$360^{\circ}$

Ruotando il mobile device lutente può esplora

LIVELLO 2

Utilizzando il touch screen lutente è in grado di esplorare

LIVELLO 3

$360^{\circ}$ VR (wearable technology)

Lutente non ha diffcoltà ad indossare il visore, ac

esplorare in modo immersivo lambiente simulato
allintemo del quale accede ai diversi contenuti.

\section{I diversi livelli di interattività}

I disturbi dello spettro autistico non si curano, durano per tutta la vita e sono difficilmente catalogabili. In altri termini spesso non si riesce a stabilire una relazione diretta con il livello e le manifestazioni e, quindi, con la capacità del soggetto ad interagire più o meno con le tecnologie. Sono stati, quindi, individuati diversi livelli di interattività:

- livello 0: l'utente non è in grado di interagire, quindi assisterà ad un normale video opportunamente predisposto per simulare una data situazione in uno spazio ipotetico. II video potrà essere accompagnato o meno da un audio che potrà essere attivato e disattivato;

- livello I: l'utente riesce ad interagire quindi potrà esplorare l'immagine panoramica semplicemente ruotando il mobile device e attivando i contenuti (video modeling) connessi all'immagine;

- livello 2: l'utente ha una maggiore capacità di interazione e quindi riesce ad esplorare la panoramica $360^{\circ}$ utilizzando il touch screen attivando in modo analogo i contenuti connessi all'immagine;

- livello 3: l'utente non ha difficoltà ad indossare il visore, ad esplorare in modo immersivo l'ambiente simulato all'interno del quale accede ai diversi contenuti. In questo caso si prevede la possibilità di sperimentare anche l'utilizzo di video modeling utilizzando video a $360^{\circ}$. Poiché l'utilizzo della wearable technology non è sempre possibile, perché in molti casi la visione immersiva può creare problemi e quindi non può essere utilizzata, abbiamo prestato particolare attenzione proprio sulla possibilità di consentire all'utente la possibilità di scegliere il livello di interattività.

\section{Deliverable di presentazione e test - INTERFACCEUSER FRIENDLY}

TOUCH SCREEN
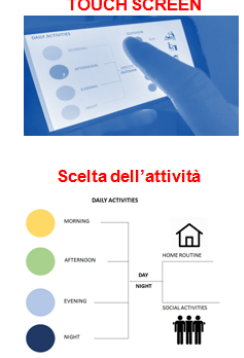

Scelta del livello di interattivita If淍 (1) (9)

䉼

Q4.

\section{Prototipo digitale - moch-up} Esplorazione dello
spazio simulato

LIVELLO $1360^{\circ}$ Ruotando il mobile device lutuente può esplorare
limmagine panoramica acoede ai contentiti utilzzan funzioni touch screen

LIVELLO $2360^{\circ}$ Utilizando il touch screen lutente in grado di esplorare lo spazio simulato accede ai contenuti

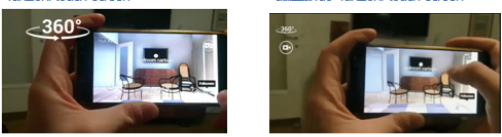

LIVELLO $3360^{\circ} \mathrm{VR}$ (wearable technology) Lutente non ha difficotà ad indossare i visore, ad esplorare lambiente simulato
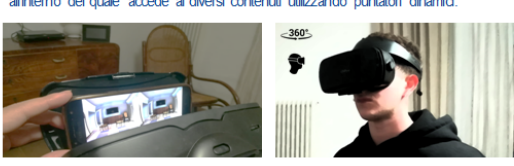

VIDEO MODELING
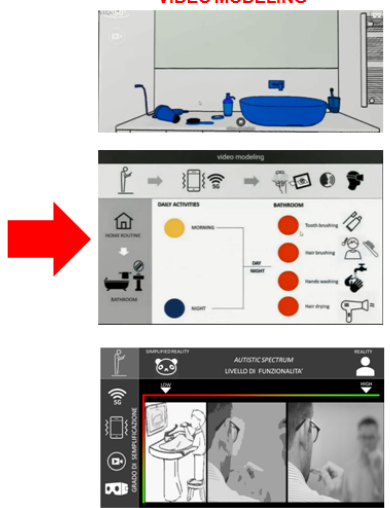

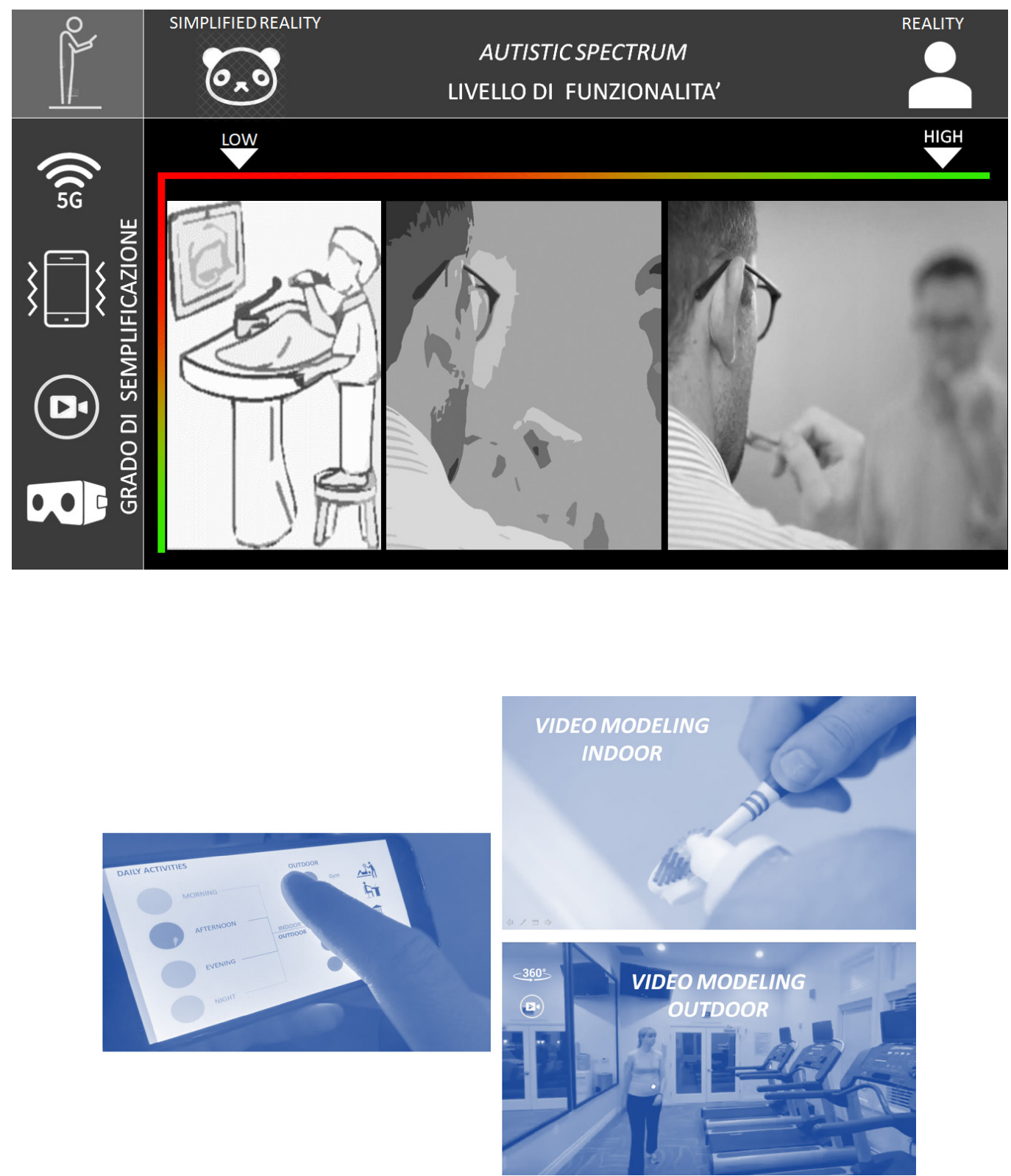

\section{Conclusioni e futuri sviluppi}

La ricerca in corso dimostra come gli ambiti di applicazione delle nostre ricerche possano essere vari e possano arricchirsi dallo scambio e dalla contaminazione con altri settori disciplinari.

Un'altra fondamentale azione congiunta è la ricerca di fonti di supporto alla ricerca pubblici o privati, e lo studio di meccanismi che consentano l'autosostentamento del progetto, sono oggetto di analisi e di studio soluzioni che prevedono un minimo contributo per il download dell'app una volta concluso il periodo di sperimentazione. Tali fondi dovranno essere vincolati alla manutenzione dell'app, ad eventuali aggiornamenti e allo sviluppo di una versione in lingua inglese da diffondere tramite network. Inoltre, la possibilità di inserire nuovi situation games, realizzati con la regia di esperti ASD, consentirà di implementare l'app rendendola sempre più versatile. 


\section{Note}

[I] Gli ideatori del sistema PECS sono Lori A. Frost e Andrew S. Bondy. II PECS è stato sviluppato nel 1994, all'interno del Delaware Autistic Program, il programma di scuole pubbliche per soggetti autistici più ampio degli Stati Uniti.

[2] Fonte:Wikipedia.

[3] Grazie all'aiuto dell'Autism Society of Boulder County (ASBC) SketchUp ha lanciato Project Spectrum e Progetto iSTAR, attività il cui scopo è connettere le comunità e gli individui coinvolti nella gestione dei disturbi dell'autismo con SketchUp.

[4]Vedi: <https://blog.sketchup.com/article/strengths-autism-shine-3d>.

[5] Vedi: <https://www.archdaily.com.br/br/920420/plugin-gratuito-permite-exportar-imagens-em-360-degrees-do-sketchuppara-plataforma-de-realidade-virtual>.

[6]Vedi: <https://3dwarehouse.sketchup.com/>

[7] Vedi: <https://3dwarehouse.sketchup.com/>.

\section{Riferimenti bibliografici}

Anderson Kristy A., Sosnowy Collette, Kuo Alice A., Shattuck, Paul T. (20 I 8). Transition of Individuals with Autism to Adulthood: A Review of Qualitative Studies. In Pediatrics. April 2018, I4I (Supplement 4).

Bondy Andy, Frost Lori (1994). The Picture Exchange Communication System. In Focus on Autism and Other Developmental Disabilities. 9(3), pp. 1 - 19.

Kandalaft Michelle R. et al. (2013). Virtual Reality Social Cognition Training for Young Adults with High-Functioning Autism. In JAutism Dev Disord. 2013 Jan; 43(1): 34-44.

Maskey Jean M. et alii (2019). A Randomised Controlled Feasibility Trial of Immersive Virtual Reality Treatment with Cognitive Behaviour Therapy for Specific Phobias in Young People with Autism Spectrum Disorder. In J Autism Dev Disord. 2019 May, 49(5), pp. 1912-1927.

Perugini Federico (2019). Esempi di utilizzo della Comunicazione Aumentativa e Alternativa. Cooperativa sociale progetto crescita onlus: <https://www.progettocrescita.it/cose-la-comunicazione-aumentativa-e-alternativa/>.

Rivarola Aurelia (2009). Comunicazione Aumentativa e Alternativa. Milano: Centro Benedetta D'Intino Onlus.

Smith Matthew J. et al. (2014). Virtual reality job interview training in adults with autism spectrum disorder. In J Autism Dev Disord. 2014 Oct; 44(I0): 2450-63.

Wright Cheryl et alii (20I I). SketchUp: A Technology Tool to Facilitate Intergenerational Family Relationships for Children with Autism Spectrum Disorders (ASD). In Family \& Consumer Science. Special Issue: Influence of Technology on Individuals and Families. Vol. 40. Issue 2, pp. I 35- I 49.

\section{Autori}

Mara Capone, Università di Napoli Federico II, mara.capone@unina.it,

Emanuela Lanzara, Università di Napoli Federico II, emanuela.lanzara@unina.it

Per citare questo capitolo: Capone Mara, Lanzara Emanuela (2020). Simulare per RI_Connettere.VR per i disturbi dello spettro autistico/Simulation for RE_Connecting.VR for autism spectrum disorders. In Arena A., Arena M., Brandolino R.G., Colistra D., Ginex G., Mediati D., Nucifora S., Raffa P. (a cura di). Connettere. Un disegno per annodare e tessere. Atti del $42^{\circ}$ Convegno Internazionale dei Docenti delle Discipline della Rappresentazione/Connecting. Drawing for weaving relationships. Proceedings of the 42 th International Conference of Representation Disciplines Teachers. Milano: FrancoAngeli, pp. $1860-1879$. 


\title{
Simulation for Re_Connecting. VR for Autism Spectrum Disorders
}

\author{
Mara Capone \\ Emanuela Lanzara
}

Abstract

ASD disorders are complex neurobehavioral syndromes: traveling, crowded places, medical examination, approaching a peer, going to university, are just some examples that can generate anxiety conditions by disconnecting an individual from real life.

The main goal of this research activity is to experiment innovative technologies to encourage relational and functional exchanges between autistic and neurotypical people and to improve their individual autonomy to manage daily life and social communication especially during developmental and adulthood age.

This activity is experimenting an app created by a multidisciplinary team made up of experts: neuropsychiatrists, clinical psychologists, computer scientists and architects.

This experimental app simulates spaces and situations, simplifies the visualization of environments, and customizes them through different levels of complexity and interactivity to facilitate users with different levels of intellectual and linguistic ability.

Different ways of simplifying simulated space, communication and interaction directly involve specific skills of scientific-disciplinary sector of representation about creation of simple video (level 0), $360^{\circ}$ panoramas (levels I and 2),VR experimentations and wearable technology.

Keywords

VR Virtual Reality, autism, user experience, immersive images, video modelling, wearable technology.

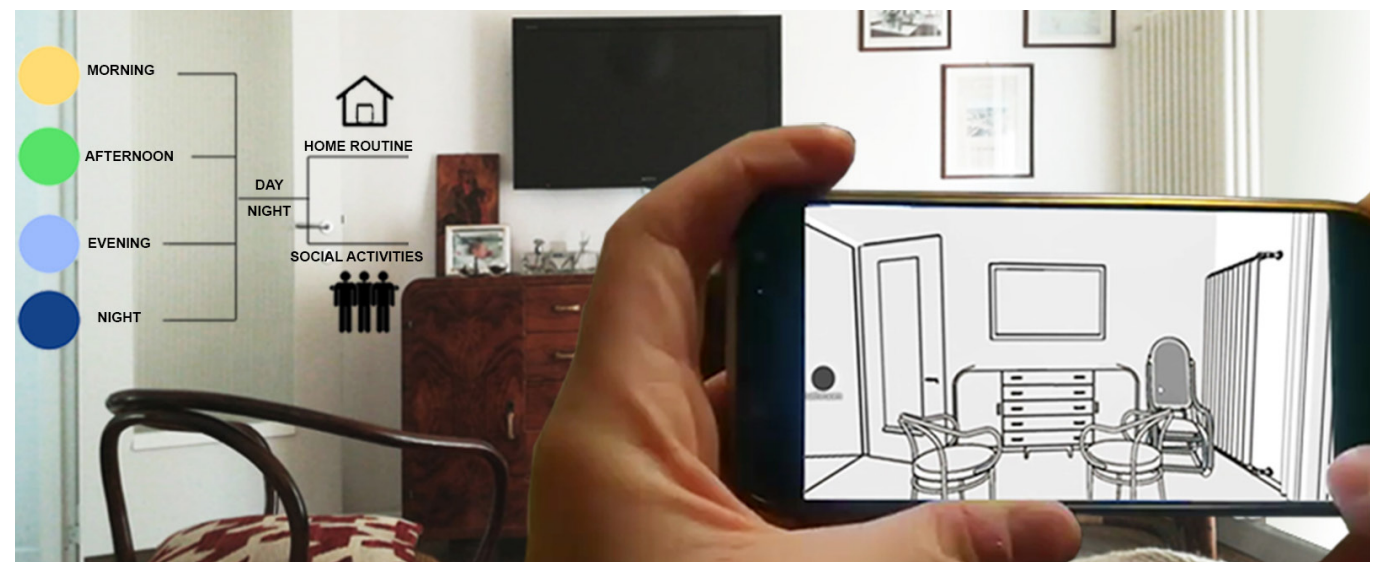




\section{Introduction}

ASD disorders are complex neurobehavioural syndromes, frequently associated with comorbidities. About $50 \%$ of ASD adults experience everyday anxiety levels. Recent studies [Kristy 20 I 8] support the importance to favour person-ambient adaptation in ASD subjects. VRE has been successfully used to improve various skills [Maskey 2019], social understanding [Kandalaft et al. 20 I3], job interview [Smith et al. 20 I 4] etc. It may offer an alternative to the traditional CBT exposure hierarchies. Analysis and results show and highlight how treatment outcomes are influenced by the level of person-environment adaptation. According to manifestations variability the supports should be personalized.

Traveling, staying in crowded places, making a medical examination, approaching a peer, going to university, are just some examples that can generate anxiety conditions and constitute a stumbling block in the daily life of an ASD.

Sometimes the therapeutic protocols applied to the general population are ineffective in ASD due to difficulties in imagining the object/situation to gradually move to living them. $A V R I V M$ tool allows to work on simulation bypassing this difficulty.

According to scientific studies about efficacy of $\bigvee R$ therapeutic tools, this project proposes an app for mobile devices to manage a high impact comorbidity on social, communicative and autonomous skills based on the simulation of scenarios that can be displayed with different levels of complexity (gameslabstractlreal) and interactivity (videol360 panoramasIVRlavatar) for users with different levels of intellectual and linguistic ability.

The scenes will be created according to the needs of each participant; a cognitive-behavioural psychotherapist will guide users and apply CBT techniques to work on relaxation, conditioning and reconditioning to the adverse stimulus following NICE guidelines.

Unlike existing apps, the research goal is to create an adaptable tool to variable needs of ASD adults through user experience and personalization.

This app will be the result of interdisciplinary work. A team of architects - 3D modeling and graphics experts, IT experts in interactive systems design, clinical experts in the autism spectrum and a non-profit organization to involve the participation of ASD subjects guarantees the necessary skills to define a performing tool to support specific perceptual needs of adults ASD simulating spaces and situations.
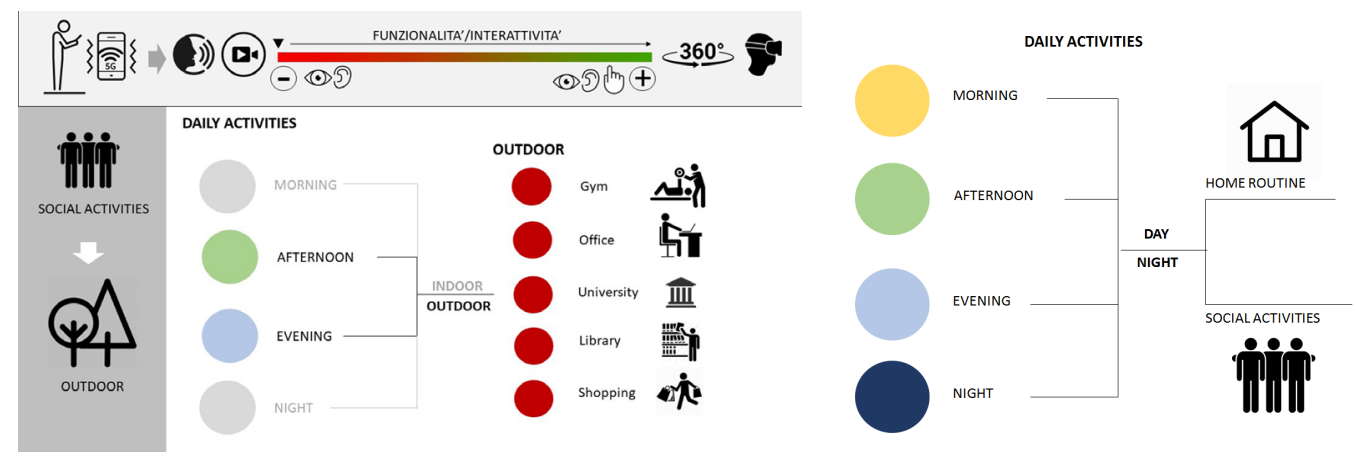

\section{Main project goals}

This adaptable, customizable, open source, user friendly app for ASD adults, families and therapists aimed at improving the person-environment adaptability, proposes the following goals: 
- promoting personal autonomy and social communication through the combination of VR (Virtual Reality) and VM (Video Modeling) by simulating spaces and situations to control exposure and anxiety stimuli in a safe and measured way;

- reach the largest number of users with an adaptable tool to supports ASD adults's needs according to their personal skills and sensitivity (Training Emotions in an Adaptable Reality System);

- reach a customizable tool to allow the possibility to switch from a generic environment to the specific one of the users;

- share the experimentation on 20 ASD adults involved by spreading the app in existing networks and promoting real and virtual meetings with stakeholders.

\section{Technologies to RE_Connect}

Virtual reality is considered an emerging effective therapeutic approach in different areas of the healthcare sector. The use of VR in ASD treatment allows to work in realistic adaptable environments according to the characteristics and capabilities of the subject.

A virtual scenario can be an environment to eliminate the pressure of real reality (RR) that ASD subjects reject. A predictive simulation is aimed at acquiring spatial awareness to prepare the subject for a specific situation in a given space.

To create the app, the most innovative technologies will be used to:

- build 3D models aimed at producing $360^{\circ}$ interactive panoramic images and videos usable with mobile devices, smartphones, tablets or VR viewers;

- visualize different levels of environmental complexity (gameslabstractlreal) and interactivity (video $1360^{\circ}$ panoramasIVRlavatar) according to different levels of functionality: minimum (video not-interactive, minimal visualizations, avatar); medium and maximum (immersive 3D visualization);

- experiment $360^{\circ}$ images to access VMs through hotspots to simulate specific actions in given times and environments;

- use photo-modeling and $360^{\circ}$ images created with special cameras or smartphones and software/app to replace generic models with real models in the app: the bathroom-my bathroom, the gym-my gym, etc.

- planning daily indoor and outdoor actions.

Adaptability, customizability are the main features of the app that make this tool flexible, easy, directly managed by families with minimal training and support. These qualities make it replicable in other rehabilitative/educational/working situations.

Connection with databases to loadVMs and the possibility to implement simulated scenarios and to customize spaces makes this app adaptable according to ASD needs.

The app allows you to customize virtual environments using images of users' real environments insert by project team.

\section{D PECS for Alternative Augmentative Communication (AAC)}

Simplification level of reality depends by different user needs in relation to his specific functional level.

In scientific field about alternative language systems, PECS (Picture Exchange Communication System) is a strategy for the Augmentative Alternative Communication (AAC) to manage of Autism Spectrum disorders [I]. Its functioning is based on the difference between 'speaking' and 'communicating', both activities aimed at connecting an individual with surrounding environment and people.

Communication in general allows a wide choice of alternative tools to create connections [Blondy et al. 1994].

In summary, knowledge and techniques about this communication strategy increase communication skills and facilitate the approach of users with difficulties in using transmission 
channels that we could define as more immediate, such as oral and written language [Rivarola 2009].

This alternative strategy is called augmentative because it does not replace natural communication methods but it is aimed at increasing them with different forms of communication, including graphics [2].

As a symbolic language, it recalls different tools and methodologies of representation.

Fig. 3. Example of CAA "widget", ARASAAC and BLISS images. The cards show different levels of simplification and codifcation of reality (Perugini 2019). The symbols can be used by digital devices or printed cards and collected in booklets.
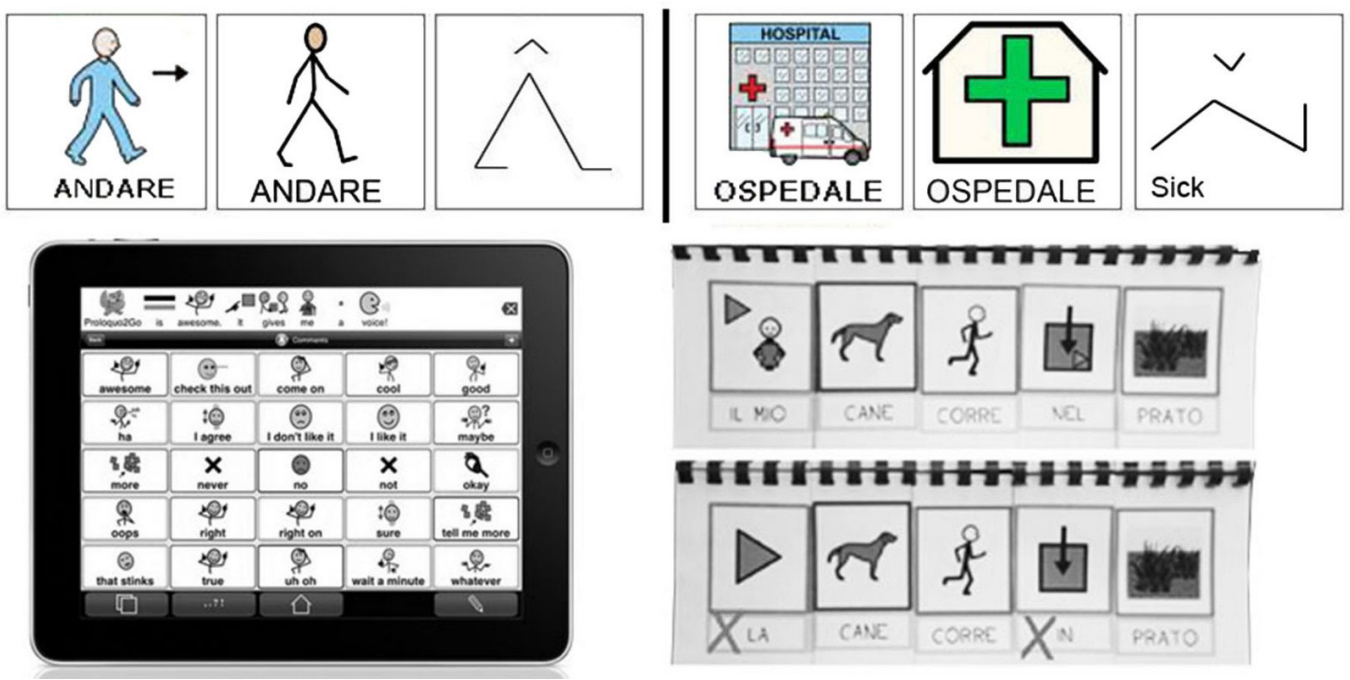

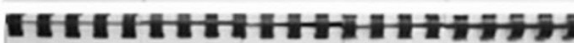

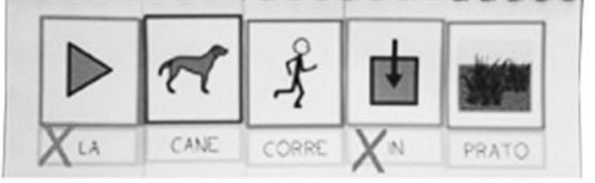

This communication system provides numerous illustrated cards collected in a personal portable booklet useful to approach different contexts of real domestic and social life. According to the same approach, virtual space allows to increase the communicative dimension of this tool composed of graphic symbols on paper or on digital supports by transform a two-dimensional code into navigable three-dimensional images, objects and contexts. Three-dimensionality allows the user to immerse himself in an interactive and predictive activities and places, alone or with other participants.

The ideal three-dimensional environment proposed in this contribution is an implementable $V R$ application designed for mobile devices (smartphones, tablets etc.). It collects videos and simulations equivalent to simplified two-dimensional symbols. The object is not a static representation of an action but a model of a dynamic virtual space that allows you to simulate actions within an alternative perceptive reality more accessible to a user with different interaction skills. This app is a portable collection of tutorials to deal daily actions and environments.

The cognitive and communicative characteristics of the user require different degrees of complexity: the power of these three-dimensional exemplary models lies in providing the user with a compatible tool whose setting and operation correspond to their specific possibilities or abilities.

Therefore, just as the CAA system provides an explanatory icon for each planned activity [3], in the same way the app will provide a hotspot icon to activate different simplification styles of a space, environment switch or video modeling of a given activity. A good balance between completeness of information and degree of simplification is important in the simplification process. 


\section{SketchUp: cartoon styles to develop a 3D PECS system}

To model environments of a domestic home or common meeting places (school, library, gym, etc.) we chose SketchUp as modeling tool. Important experiences in this field [4] show that for some users, especially non-verbal users, SketchUp is an effective communication tool to share thoughts through images and reaching important educational and working activities and goals.

Scientific literature [Wright et al. 20I I] documents positive results about the use of this CAD interface by autistic users, including experiences in modeling simple two-dimensional objects (circles, squares), development of stronger interpersonal relationships and greater confidence allowed by the use of a platform suitable to support patients' latent abilities [5].

Therefore, this communication project chooses SketchUp's interface and intuitive approach as optimal tool for a possible independent use or to develop an educational project that mainly involves users with a high level of functionality.

The cartoon or sketch effects of its visualization styles (Realistic, Shaded and Hidden Line) resume graphicalization and gradual simplification of the different systems used for PECS cards. The goal of the app is to transform these 2D actions into 3D objects and environments through representation methods based on a scientific and consolidated approach.
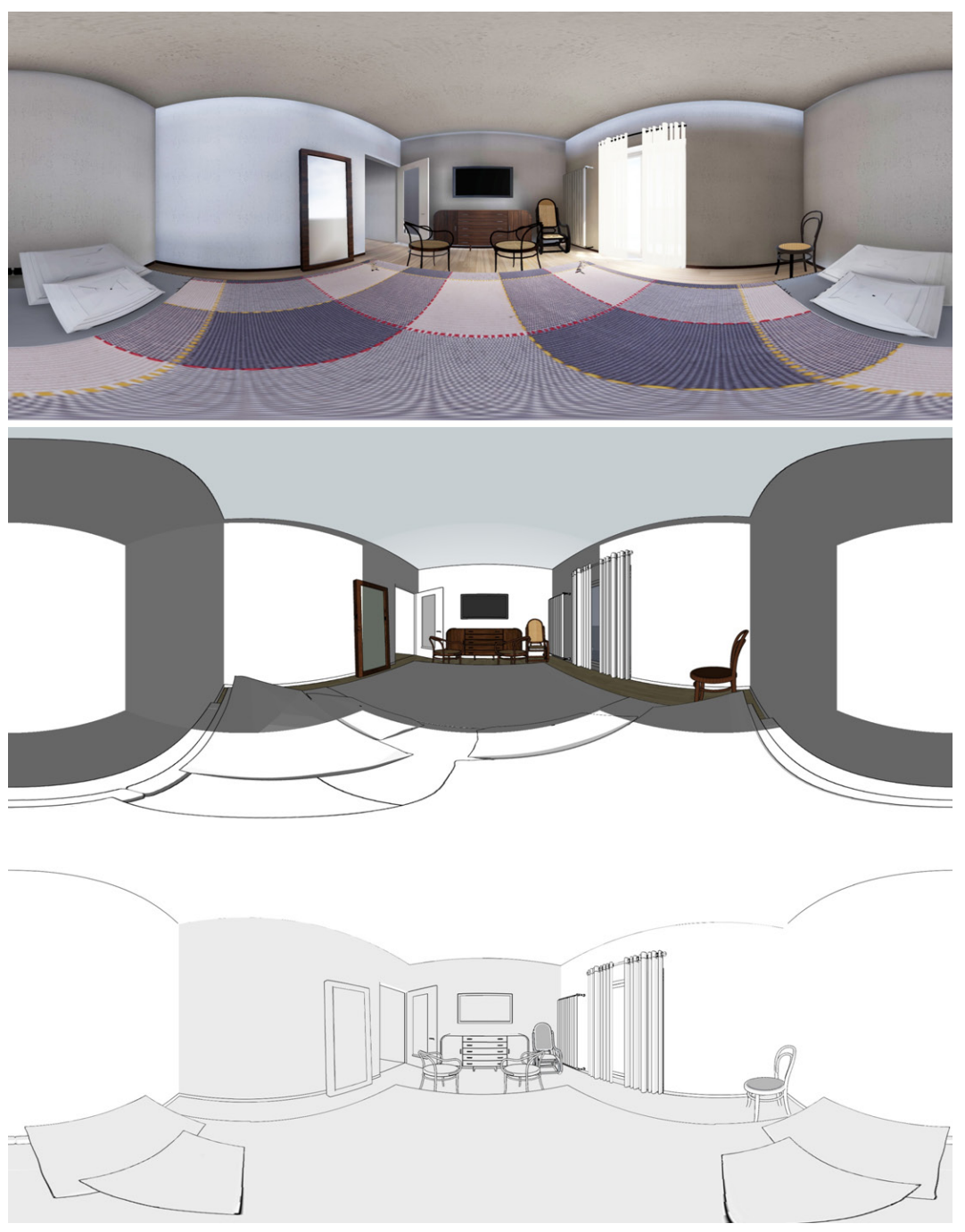


\section{Predictive representation systems: from $360^{\circ}$ render to video modeling}

Special fast and intuitive plug-ins (e.g. Tour Facil [6]) allow to quickly export $360^{\circ}$ renderings - cubic render of environments modeled in SketchUp using predefined visual styles listed in the previous paragraph.

These tools do not need to set a perspective reference system (position of the point of view and visual field) to generate $360^{\circ}$ rendering. The process starts framing an initial scene: a special plug-in allows to extract six photos generated by the projection of the environment on the six faces of a cube. Finally, it is necessary to upload these images on a special platform to automatically generates a cubic photo or render.

To generate a cubic photo it is necessary to combine a series of six square images with same size, same name but different suffix (e.g. test camera.jpg-Front; test camera.jpgDown, etc.) to indicate their mutual cubic position (cube maps).

Appropriate VR apps (open source or commercially available) use panoramas or cubic photos to simulate walking in consecutive environments with different levels of simplification.

Cubic renders can be navigate on a display or using a viewer or virtual glasses: the choice of device depends on the level of functionality of the ASD user.
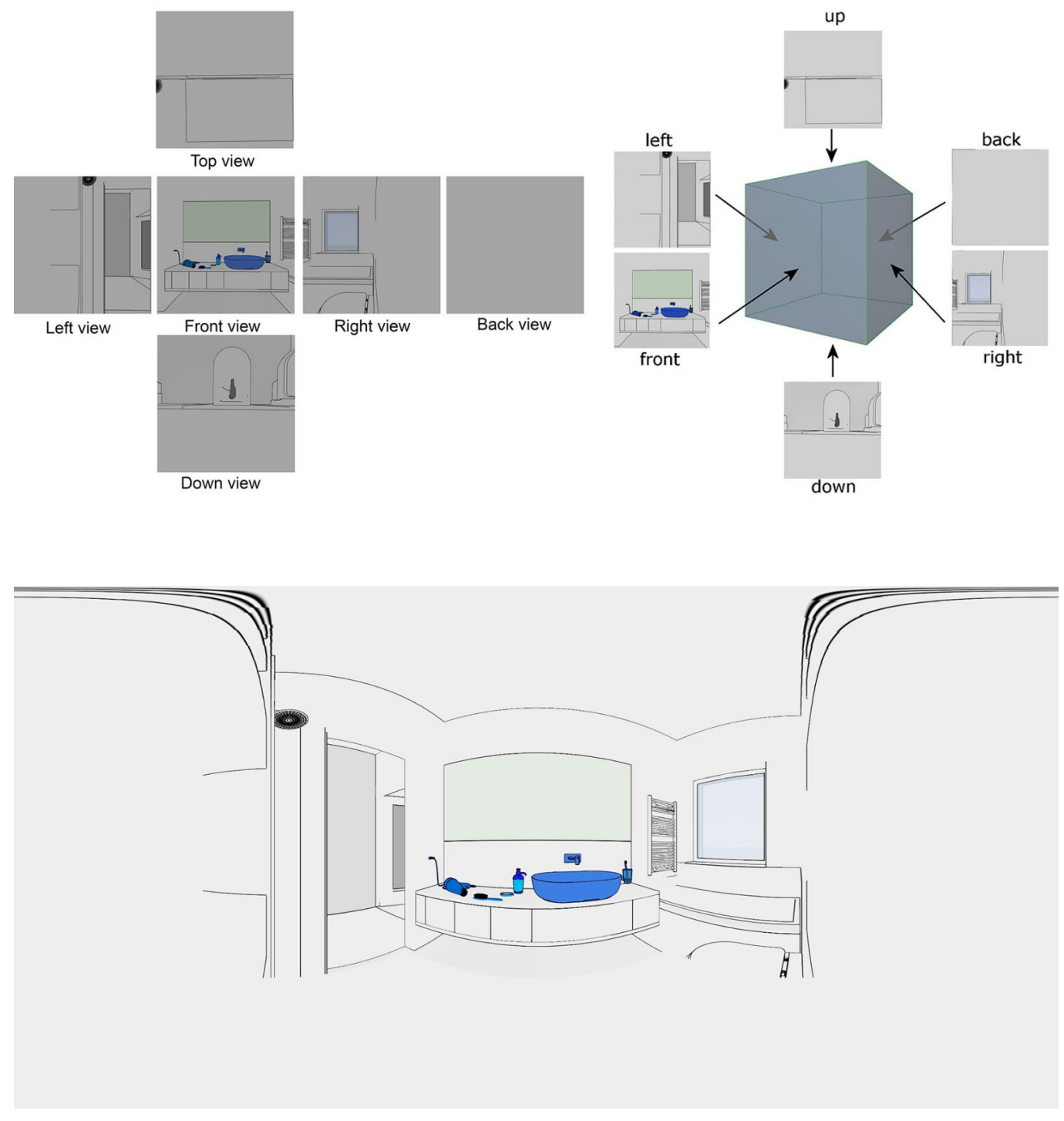
The environments sequence can be linked to specific planned daily activities (indoor and outdoor, during daytime, afternoon or evening). By clicking on a series of hotspots suitably positioned in $360^{\circ}$ views it is possible to activate the scene change. In addition, hotspots also allow you to switch from one visual style to another.

A rich and implementable library of 3D objects available in Sketchup 3D Warehouse platform [7] allow to characterize environments and can be connected to hotspots becoming real buttons to start video modeling about action intuitively connectable to the selected object (eg: phon object clickhotspot = video modeling how to dry your hair).

\section{UX DESIGN}

II progetto dell'User Experience prevede una fase di sperimentazione sull'App prototipo con l'obiettivo di definire livelli di semplificazione e di interazione con i contesti simulati adeguati ai diversi livelli di funzionalità.

\section{SEMPLIFICARE}

L'utente può scegliere la modalità di visualizzazione

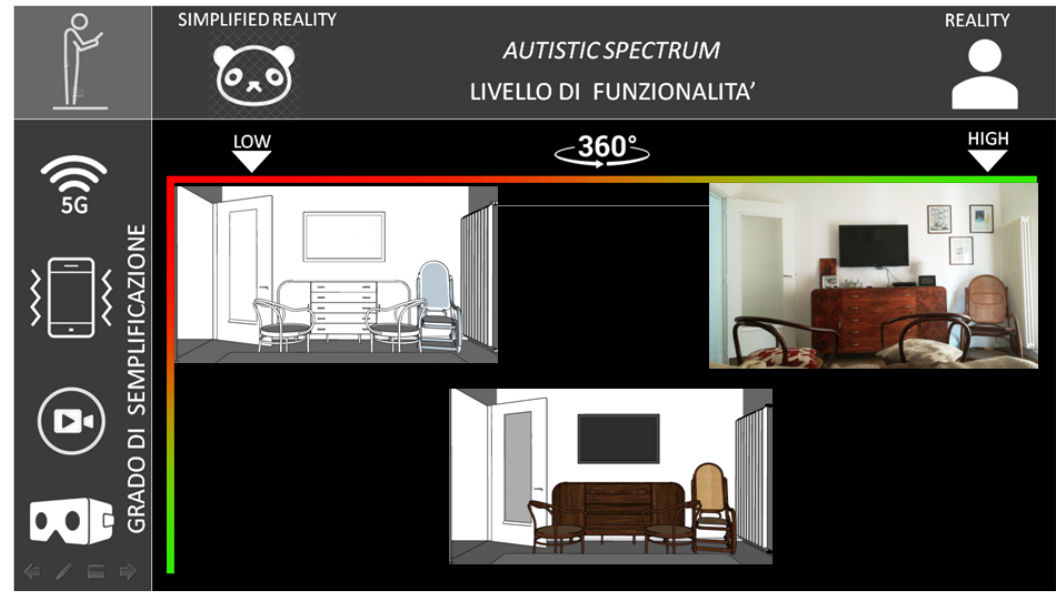

\section{UX DESIGN}

II progetto dell'User Experience prevede una fase di sperimentazione sull'App prototipo con l'obiettivo di definire livelli di semplificazione e di interazione con i contesti simulati adeguati ai diversi liveli di funzionalità. SEMPLIFICARE

L'utente può passare da una modalità di visualizzazione all'altra anche durante l'esplorazione utilizzando la funzione touch screen o il sistema di puntatore per VR
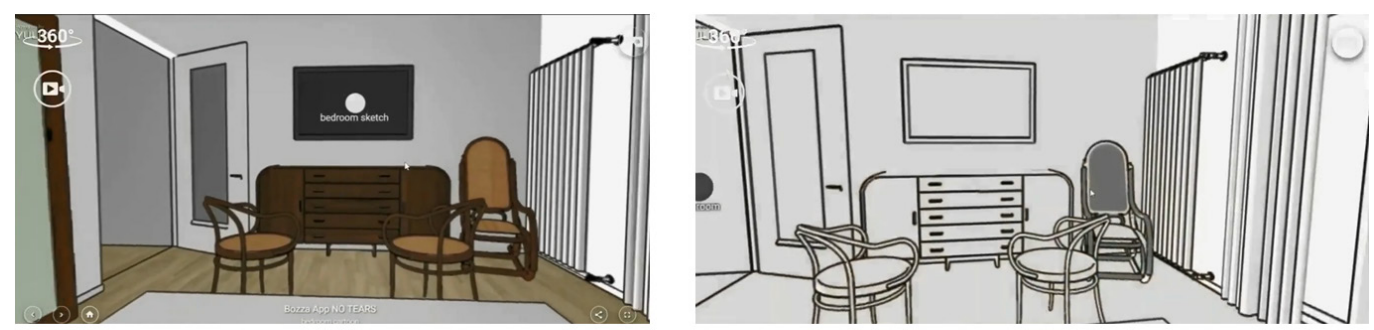
Fig. 8. Different levels of interactivity.
PERSONALIZZARE IL LIVELLO DI INTERATTIVITA

L'App prevede diversi livelli di complessità e interattività adattandosi ad utenti con differenti livelli di abilità intellettive.

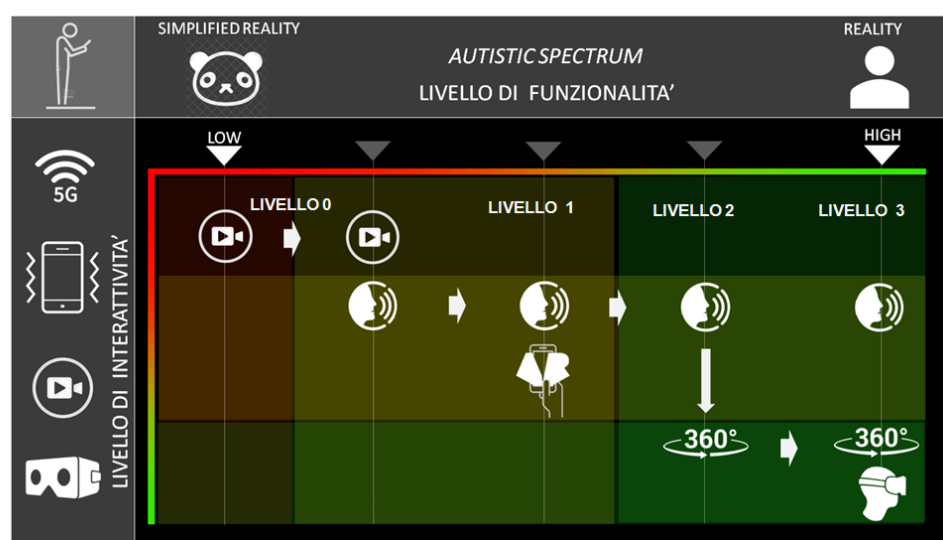

LIVELLOO 0

SOlo VIDEO AUDIO

Lutente non ein grado di i interagire autonomamente

LIVELLO 1

$360^{\circ}$

Ruotando il mobile device lutente può esplora

LIVELLO 2

Utilizzando il touch screen lutente è in grado di esplorare

LIVELLO 3

$360^{\circ}$ VR (wearable technology)

Lutente non ha diffcoltà ad indossare il visore, ac

esplorare in modo immersivo lambiente simulato
allintemo del quale accede ai diversi contentiti.

\section{Different levels of interactivity}

Autism spectrum disorders are not curable, they are lifelong and difficult to catalog. Very often it is not possible to establish a direct relationship between levels of functionality, manifestations, and interactive abilities of ASD subject regarding use of technologies. Therefore, it is possible to identify different levels of interactivity:

- level 0: the user cannot interact and will attend a normal video suitably prepared to simulate a given situation in a hypothetical space. The video may be accompanied by an audio that can be activated if useful;

- level I: the user can interact and explore the panoramic image by rotating the mobile device and activating the contents (video modeling) connected to the image;

- level 2: the user has greater interaction and can explore a $360^{\circ}$ panorama using the touch screen and activating the contents connected to the image;

- level 3: the user can wear virtual glasses to immersively explore simulated environment and he accesses various contents. In this case he can also experiment video modeling and $360^{\circ}$ video.

The use of wearable technology is not always possible: in many cases immersive vision can create problems and cannot be used. This project pays particular attention to the possibility of choosing a specific level of interactivity.

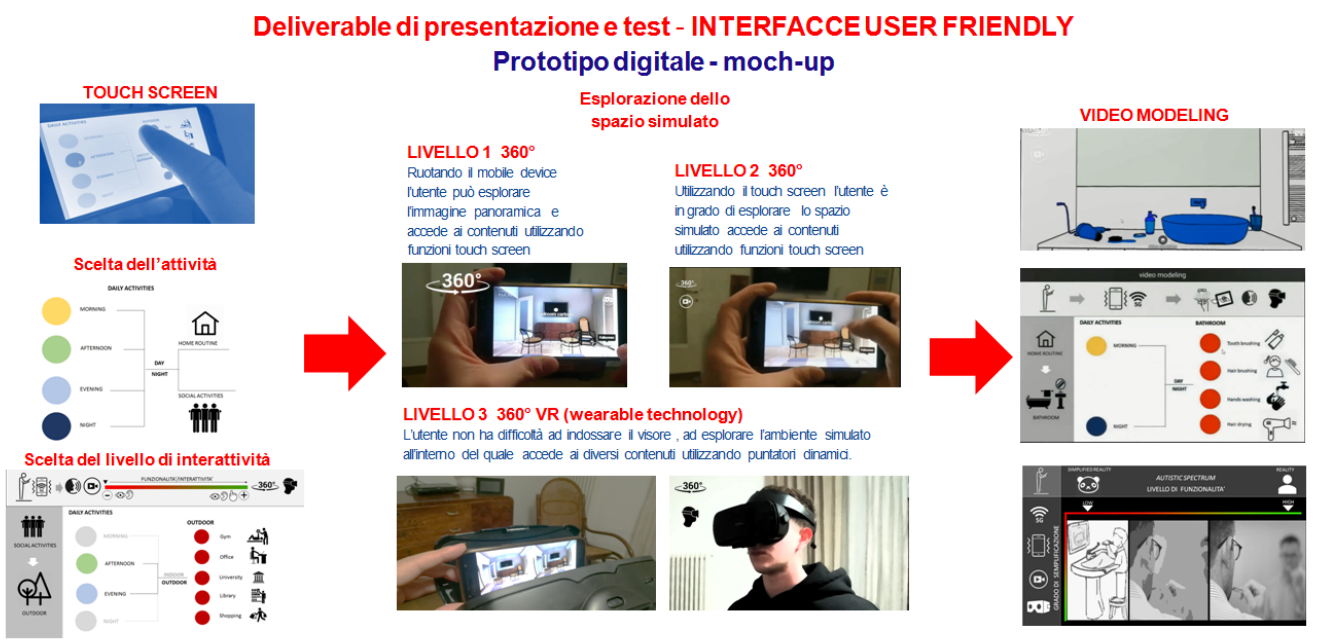



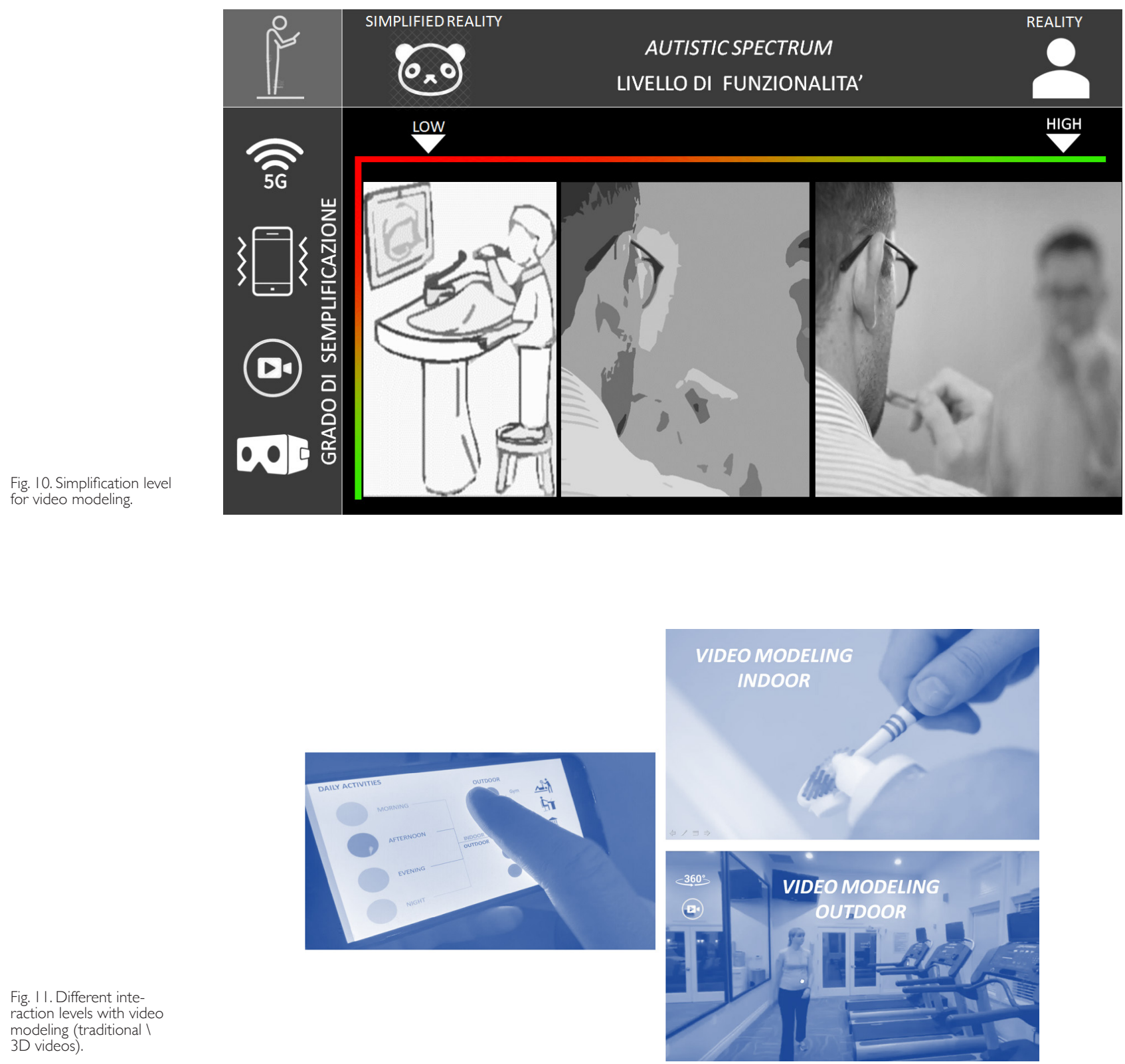

\section{Conclusions and future developments}

This research work in progress demonstrates how applications of representation disciplines can be various and they can be enriched by exchange and contamination with other disciplinary sectors.

Particular attention may be paid to the design or use of interfaces that facilitate ASD users in independent modeling activity and to the development of educational programs. Another goal is to research public or private sources to support this research project and to allow for self-support of the project: solutions for a minimal contribution to download the app once the trial period is over are subject to analysis and study.

These funds must be tied to the maintenance of the app, its updates and to develop an English version.

In addition, the possibility of inserting new situation games designed with ASD experts will allow to implement the app making it a growing and versatile tool. 


\section{Notes}

[I] Lori A. Frost and Andrew S. Bondy are the creators of PECS. This system was developed in 1994 within the Delaware Autistic Program, the largest public-school program for autistic subjects in the United States.

[2] Source: Wikipedia.

[3] See: <https://www.progettocrescita.it/cose-la-comunicazione-aumentativa-e-alternativa/>.

[4] Autism Society of Boulder County (ASBC) and SketchUp have launched Project Spectrum and iSTAR Project, activities to connect communities and individuals involved in the management of autism disorders using SketchUp.

[5] See: <https://blog.sketchup.com/article/strengths-autism-shine-3d>.

[6] See: <https://www.archdaily.com.br/br/920420/plugin-gratuito-permite-exportar-imagens-em-360-degrees-do-sketchuppara-plataforma-de-realidade-virtual>.

[7] See: <https://3dwarehouse.sketchup.com/>.

\section{References}

Anderson Kristy A., Sosnowy Collette, Kuo Alice A., Shattuck, Paul T. (2018). Transition of Individuals with Autism to Adulthood: A Review of Qualitative Studies. In Pediatrics. April 2018, I4I (Supplement 4).

Bondy Andy, Frost Lori (1994). The Picture Exchange Communication System. In Focus on Autism and Other Developmental Disabilities. 9(3), pp. 1 - 19.

Kandalaft Michelle R. et al. (2013). Virtual Reality Social Cognition Training for Young Adults with High-Functioning Autism. In JAutism Dev Disord. 2013 Jan; 43(1): 34-44.

Maskey Jean M. et alii (2019). A Randomised Controlled Feasibility Trial of Immersive Virtual Reality Treatment with Cognitive Behaviour Therapy for Specific Phobias in Young People with Autism Spectrum Disorder. In J Autism Dev Disord. 2019 May, 49(5), pp. 1912-1927.

Perugini Federico (2019). Esempi di utilizzo della Comunicazione Aumentativa e Alternativa. Cooperativa sociale progetto crescita onlus: <https://www.progettocrescita.it/cose-la-comunicazione-aumentativa-e-alternativa/>.

Rivarola Aurelia (2009). Comunicazione Aumentativa e Alternativa. Milano: Centro Benedetta D'Intino Onlus.

Smith Matthew J. et al. (2014). Virtual reality job interview training in adults with autism spectrum disorder. In J Autism Dev Disord. 2014 Oct; 44(10): 2450-63.

Wright Cheryl et alii (20I I). SketchUp: A Technology Tool to Facilitate Intergenerational Family Relationships for Children with Autism Spectrum Disorders (ASD). In Family \& Consumer Science. Special Issue: Influence of Technology on Individuals and Families. Vol. 40. Issue 2, pp. I35- I 49.

\section{Authors}

Mara Capone, Università di Napoli “Federico II", mara.capone@unina.it,

Emanuela Lanzara, Università di Napoli "Federico II", emanuela.lanzara@unina.it

To cite this chapter: Capone Mara, Lanzara Emanuela (2020). Simulare per RI_Connettere.VR per i disturbi dello spettro autistico/Simulation for RE_Connecting. VR for autism spectrum disorders. In Arena A., Arena M., Brandolino R.G., Colistra D., Ginex G., Mediati D., Nucifora S. Raffa P. (a cura di). Connettere. Un disegno per annodare e tessere. Atti del $42^{\circ}$ Convegno Internazionale dei Docenti delle Discipline della Rappresentazione/Connecting. Drawing for weaving relationships. Proceedings of the 42th International Conference of Representation Disciplines Teachers. Milano: FrancoAngeli, pp. 1860 - 1879 